\title{
A MAPPING ASSOCIATED TO $h$-CONVEX VERSION OF THE HERMITE-HADAMARD INEQUALITY WITH APPLICATIONS
}

\author{
M. Rostamian Delavar and M. De La Sen
}

Abstract. This paper deals with a real mapping $L(t)$ related to the $h$-convex version of the Hermite-Hadamard inequality. In special cases some generalized form of the Hermite-Hadamard inequality for convex functions are obtained. Also, as an application some inequalities for special means are given.

Mathematics subject classification (2010): 26D15, 26A51, 52A01.

Keywords and phrases: $h$-convex function, Hermite-Hadamard inequality, special means.

\section{REFERENCES}

[1] W. W. BRECKNER, Stetigkeitsaussagen für eine Klasse verallgemeinerter konvexer funktionen in topologischen linearen Räumen, Publ. Inst. Math. 23 (1978), 13-20.

[2] S. S. Dragomir, D. S. MilošEvić And And J. SÁndor, On some refinements of Hadamard's inequalities and applications, Univ. Belgrad, Publ. Elek. Fak. Sci. Math., 4 (1993), 21-24.

[3] S. S. Dragomir And C. E. M. Pearce, Selected topics on Hermite-Hadamard inequalities and applications, RGMIA Monographs, Victoria University, 2000. (online: http://ajmaa.org/RGMIA/monographs.php/)

[4] S. S. Dragomir, J. PeČArić, L. E. Persson, Some inequalities of Hadamard type, Soochow J. Math. 21 (1995), 335-341.

[5] E. K. Godunova, V. I. Levin, Neravenstva dlja funkcii širokogo klassa, soderžaščego vypuklye, monotonnye i neko-torye drugie vidy funkcii, in: Vyčislitel. Mat. i. Mat. Fiz. Mežvuzov. Sb. Nauč. Trudov, pp. 138-142, MGPI, Moskva, 1985.

[6] J. PeČarić, F. Proschan, Y. L. Tong, Convex Functions, Partial Orderings and Statistical Applications, Academic Press, Inc., 1992.

[7] M. Rostamian Delavar And M. De La Sen, Some generalizations of Hermite-Hadamard type inequalities, SpringerPlus, 5:1661 (2016).

[8] M. Rostamian Delavar and S. S. Dragomir, On $\eta$-convexity, Math. Inequal. Appl. 20 (2017), 203-216.

[9] M. Rostamian Delavar, M. Kian, On extension of an inequality including Arithmetic and Logarithmic means via generalized Hermite-Hadamard inequality, submitted.

[10] M. Z. Sarikaya, A. Saglam and H. Yildirim, On Some Hadamard-Type Inequalities for $h$ Convex Functions, Journal of Mathematical Inequalities. 2(3) (2008), 335-341.

[11] S. VarošAnec, On h-convexity, J. Math. Anal. Appl. 326 (2007), 303-311. 